\title{
Chemical Reactions and Dust Destruction in Protoplanetary Accretion Disks
}

\author{
Frédéric Finocchi ${ }^{1}$, Hans-Peter Gail ${ }^{1}$, Wolfgang J. Duschl ${ }^{1,2}$, and \\ Werner M. Tscharnuter ${ }^{1}$ \\ 1 Institut für Theoretische Astrophysik, Universität Heidelberg, \\ Tiergartenstr. 15, D-69121 Heidelberg, Germany \\ 2: Max-Planck-Institut für Radioastronomie, Auf dem Hügel 69, \\ D-53121 Bonn, Germany
}

\begin{abstract}
A nonequilibrium calculation for the chemistry in a classical protoplanetary accretion disk is presented. Slow radial particle transport moves grains from the cold outer regions of a protoplanetary accretion disk into its warm central part where grains are destroyed. We consider the destruction processes for the silicate and carbon dust component and follow the chemical composition of the gas as a function of the radial distance from the protostar. The main result of this calculation is the presence of huge amounts of methane at a distance of $\sim 1$ AU from the protostar as product of the carbon dust destruction. It is very likely that more complex organics also are present in this region.
\end{abstract}

\section{Introduction}

Chemical processes (e.g., molecules and dust reactions) play an important role for the structure and evolution of protoplanetary accretion disks. The chemical reactions, together with dust formation and destruction, determine the abundance of the absorbers and, thus, the opacity of the mixture of gas and dust, and the gas temperature. Temperature changes due to opacity changes, in turn, influence the rates of the chemical processes and the further chemical evolution of the system. This interplay between chemistry, opacity and temperature is of crucial importance and decides about the stability or instability of the disk, i.e., decides whether a disk may exist at all. Currently, limit cycle instabilities are under discussion as possible causes for the luminosity variations of certain classes of variable stars.

\section{Model assumptions}

The evolution in time and space of accretion disks as well as their chemical evolution are of fundamental importance for understanding the formation of planetary systems in general, and of our Solar System in particular. 


\subsection{Gas phase chemistry}

The evolution of the number density $n_{i}$ of each species $i$ is determined by the continuity equation

$$
\partial_{t} n_{i}+\partial_{\vec{x}}\left(n_{i} \vec{v}\right)=R_{i}
$$

( $v$ : gas velocity). Turbulent diffusion in a viscous accretion disk is not yet taken into account. In the present model calculation the continuity equation is solved in a comoving reference frame. In this case the set of rate equations is reduced to a system of ordinary differential equations.

For the rate coefficients $R_{i}$ we take into account binary reactions between gas phase species, some ternary reactions to allow for $\mathrm{H}_{2}$ formation in the denser parts of the disk, and surface reactions with dust particles to allow for dust particle destruction and growth. The corresponding rate coefficients for the gas phase reactions are

$$
R_{i}=\sum_{j, l} k_{i j l} n_{j} n_{l} \quad \text { and } \quad R_{i}=\sum_{j, l, m} k_{i j l m} n_{j} n_{l} n_{m}
$$

The rate coefficients for gas phase reactions are approximated by the standard Arrhenius form

$$
k=A T^{\beta} \exp \left(-T_{0} / T\right) .
$$

Currently, we consider only neutral-neutral reactions of $\approx 80$ atomic and molecular species of the most abundant elements $\mathrm{H}, \mathrm{C}, \mathrm{N}, \mathrm{O}, \mathrm{Mg}$, and $\mathrm{Si}$, and $\approx 450$ chemical reactions between these species. The chemical network is nearly identical with that given by Mitchell (1984). Several rate coefficients have been updated using data from Baulch et al. (1992).

Additional rate terms account for the injection of matter into the gas phase when ice mantles evaporate.

\subsection{Evaporation of dust and ice}

In order to investigate the evaporation of ice mantles and of dust particles we use the following model:

a) We assume that there exist two dust components: olivine and carbon dust. Initially all $\mathrm{Si}$ is bound in olivine and $70 \%$ of the carbon is bound in carbon dust (with the remaining $30 \%$ in the gas phase as $\mathrm{CO}$ ) according to Mathis et al. (1977). Since the thermodynamical properties of olivine seem not to be known we treat this dust component as $\mathrm{Mg}_{2} \mathrm{SiO}_{4}$.

b) At the disk's outer radius the temperatures are so low that - with the exception of $\mathrm{H}_{2}, \mathrm{H}$ and $\mathrm{N}_{2}$ - all molecular species are frozen out, especially $\mathrm{H}_{2} \mathrm{O}$ and $\mathrm{CO}$. Less abundant species like $\mathrm{CH}_{3} \mathrm{OH}$, which are frozen into the ice mantles (Pollak et al. 1994) and are liberated during their vaporization, are presently not considered. We assume that the dust grains are coated by an outer mantle of $\mathrm{CO}$ ice and an inner mantle of $\mathrm{H}_{2} \mathrm{O}$ ice. This neglects that some of the $\mathrm{CO}$ is bound into the $\mathrm{H}_{2} \mathrm{O}$ ice (Sandford \& Allamandola 1993).

c) Evaporation of carbon at $\approx 1500 \mathrm{~K}$ adds $\mathrm{C}_{i}$ molecules to the gas phase (mainly with $i=1,2,3$ ). These undergo further reactions until they finally are transformed into CO. The corresponding reactions are part of our reaction network. Silicate dust is destroyed by evaporation above $\approx 1650 \mathrm{~K}$. This mainly 
adds $\mathrm{SiO}, \mathrm{O}$, and $\mathrm{Mg}$ to the gas phase. Details of our treatment of evaporation of dust particles are given in Duschl et al. (1996).

d) We a assume a size distribution of the ensemble of dust particles as in Mathis et al. (1977). This does not agree well with the absorption properties of dust in star forming regions, where small particles seem to be less abundant than in the Mathis et al. model. The strong coupling between dust absorption and disk structure during the silicate vaporization depends, however, only on the disappearance of the large grains (see Duschl et al. 1996).

e) The process of evaporation of ice mantles and of dust is modelled by calculating the change of radius of the ice layers and the dust particles, taking properly into account the size distribution of dust grains.

\subsection{Oxidation of carbon dust}

The carbon dust can be eroded by chemical reactions with molecules from the gas phase. From flame chemistry it is known that the most efficient processes are oxidation by oxygen bearing molecular species, especially by $\mathrm{OH}$ and $\mathrm{O}_{2}$ (El-Gamal 1995). The most promising process in the protoplanetary disk is oxidation by $\mathrm{OH}$ radicals. The essential first step is (El-Gamal 1995):

$$
\operatorname{soot}_{\mathrm{N}+2}+\mathrm{OH} \rightarrow \operatorname{soot}_{\mathrm{N}}+\mathrm{HCCO}
$$

which releases two carbon atoms. The ketyl radical HCCO reacts in the gas phase according to

$$
\mathrm{HCCO}+\mathrm{H} \rightarrow \mathrm{CH}_{2}+\mathrm{CO}
$$

This converts one of the two carbon atoms immediately into CO. The subsequent reactions of the $\mathrm{CH}_{2}$ radical are described later.

\subsection{The disk model}

Currently we describe the structure of the protoplanetary disk by a semi-analytical model in the approximation of a thin, viscous accretion disk. The pressure $P$, central plane temperature $T$, vertical thickness $h$, viscosity $\nu$ and inward drift velocity $v_{s}$ are given in this model by (Duschl et al. 1996)

$$
\begin{aligned}
P & =71.65 s^{-\frac{51}{20}}\left(\frac{M}{M_{\odot}}\right)^{\frac{5}{8}}\left(M_{-7} \kappa\right)^{\frac{1}{8}}\left(\frac{1}{\mu}\right)^{\frac{1}{2}}\left[\frac{\mathrm{g}}{\mathrm{cms}^{2}}\right] \\
T & =997 s^{-\frac{9}{10}}\left(\frac{M}{M_{\odot}}\right)^{\frac{1}{4}}\left(M_{-7} \kappa\right)^{\frac{1}{4}} \quad[\mathrm{~K}] \\
\nu & =2.867 .10^{14} s^{\frac{3}{5}} M_{-7}\left[\frac{\mathrm{cm}^{2}}{\mathrm{~s}}\right] \\
h & =1.446 .10^{12} s^{\frac{21}{20}}\left(\frac{M}{M_{\odot}}\right)^{-\frac{3}{8}}\left(M_{-7} \kappa\right)^{\frac{1}{8}}\left(\frac{1}{\mu}\right)^{\frac{1}{2}} \quad[\mathrm{~cm}] \\
v_{s} & =26.94 s^{-\frac{2}{5}} M_{-7}\left[\frac{\mathrm{cm}}{\mathrm{s}}\right]
\end{aligned}
$$

The symbols have the following meanings: $s$ is the radial distance from the accreting object in AU, $M$ is the mass of the central accreting object (the Protosun), $\mathrm{M}_{\odot}$ is the solar mass, $M_{-7}$ is the accretion rate in units of $10^{-7} \mathrm{M}_{\odot} / \mathrm{yr}$, 
$\kappa$ is the mass absorption coefficient and $\mu$ is the mean molecular weight. The numbers correspond to an assumed surface mass density of $2500 \mathrm{~g} / \mathrm{cm}^{2}$ at 1 AU.

The dust opacity is calculated simultaneously with the chemical composition of the gas, the destruction processes for the dust grains, and the disk structure. The mass absorption coefficient of the dust is calculated from the model of Draine (1985). For simplicity, we currently assume that the mass absorption coefficient of the gas component is constant.

We prescribe the initial conditions for the chemistry at $s=1000 \mathrm{AU}$ and solve the equations from this point inwards. The initial composition of the gas phase is assumed to be: $10^{-5}$ of the hydrogen as free atoms, the remaining fraction not bound into molecules is $\mathrm{H}_{2}$, all nitrogen is in $\mathrm{N}_{2}$. All carbon not bound in dust particles is in $\mathrm{CO}$ and all oxygen not bound in $\mathrm{CO}$ or dust is in $\mathrm{H}_{2} \mathrm{O}$. $\mathrm{CO}$ and $\mathrm{H}_{2} \mathrm{O}$ are frozen out as ice mantles on the dust particles. Less abundant species may be present in the gas phase, in the dust grains or in their ice mantles (e.g., Pollack et al. 1994), but these are neglected because they are unlikely to be of importance for the chemistry in the warm inner regions of the disk. We use solar system abundances as given by Anders \& Grevesse (1989).

We follow the chemical evolution in a gas element which moves from larger radii towards the center of the disk with velocity $v_{s}$. During this inward drift the temperature and pressure increases resulting in changes in the chemical composition of the gas phase and of the amounts of ices and dust present. The temperature is calculated at every time step self-consistently taking into account the calculated dust opacity and an approximation for the gas opacity.

This model results in an extremely stiff system of ordinary differential equations that is coupled with a set of strongly non-linear algebraic equations for the disk structure and opacity. For the integration of the system we use a multistep, variable order backward differentiation method described in Bauer et al. (1996).

\section{Results and interpretation}

Figure 1 shows the chemical composition of the gas phase in the disk's central plane in the radial range where the carbon and silicate dust is destroyed. At larger radii (not shown here) first $\mathrm{CO}$ ice evaporates at $\approx 21 \mathrm{AU}$ where $T \approx 30 \mathrm{~K}$, and then water ice evaporates at $\approx 8 \mathrm{AU}$ where $T \approx 150 \mathrm{~K}$. The first dust component to be destroyed is the carbon dust. Between 1 and $0.5 \mathrm{AU}$, in the temperature regime between $T \approx 1000$ and $\approx 1300 \mathrm{~K}$ (see Fig. 3), the carbon is oxidized by $\mathrm{OH}$ radicals resulting ultimately in an increase of the $\mathrm{CO}$ abundance in the gas phase and a corresponding decrease of the water steam abundance. In principle, the carbon is destroyed in the protostellar disk by the process of water gas production which converts carbon into $\mathrm{CO}$ and $\mathrm{H}_{2}$.

The first steps of this process are the two reactions (4) and (5). The $\mathrm{CH}_{2}$ resulting from reaction (5) reacts with molecular hydrogen to form $\mathrm{CH}_{3}$ and $\mathrm{CH}_{4}$

$$
\mathrm{CH}_{2}+\mathrm{H}_{2} \leftrightarrow \mathrm{CH}_{3}+\mathrm{H} \quad, \quad \mathrm{CH}_{3}+\mathrm{H}_{2} \leftrightarrow \mathrm{CH}_{4}+\mathrm{H}
$$

From several possible reaction paths from this to $\mathrm{CO}$, the most efficient one in the protoplanetary disk according to the results of our model calculation seems to be the condensation reaction 


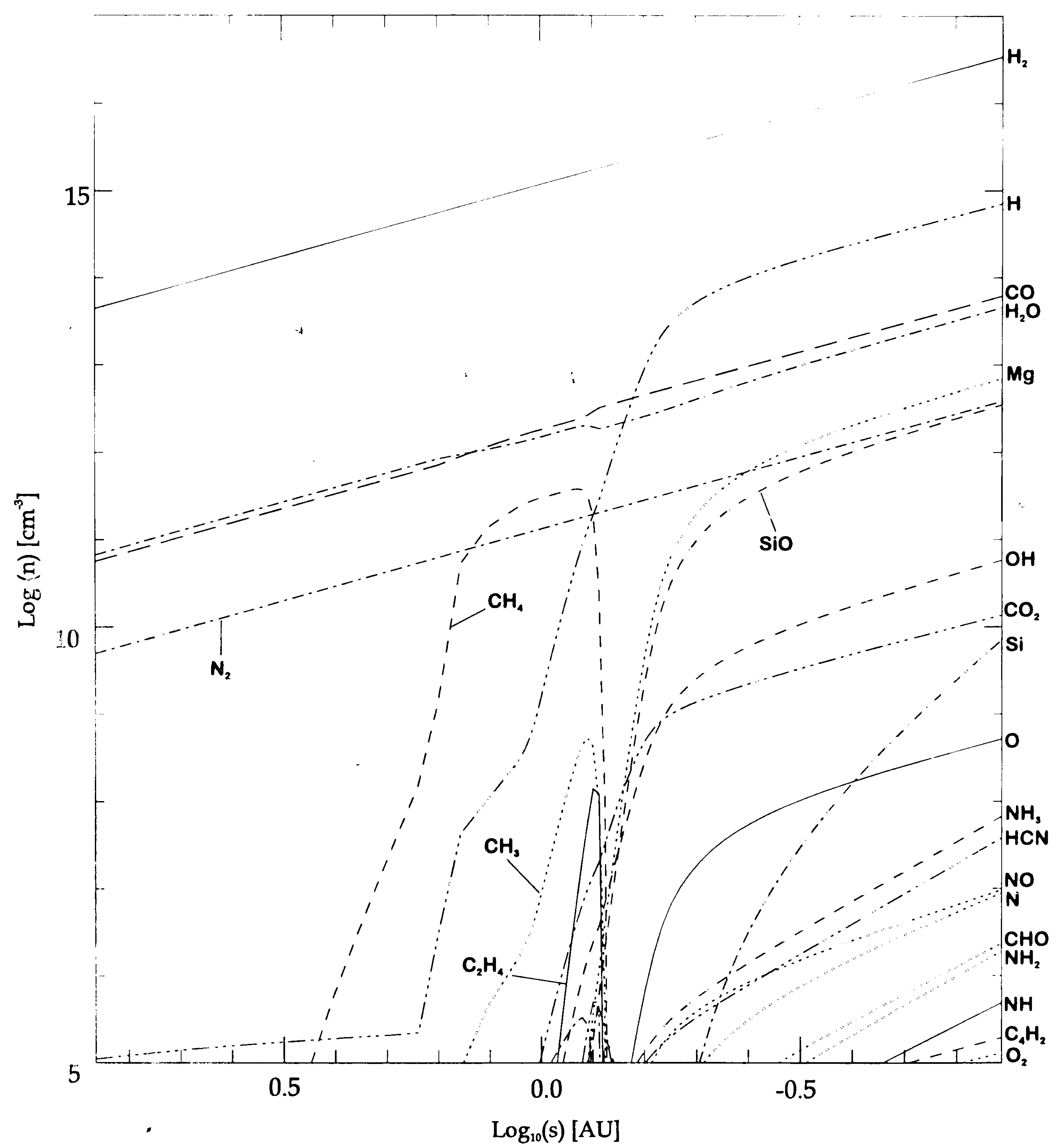

Figure 1 Chemical composition of the disk in the central plane

$$
\mathrm{CH}_{3}+\mathrm{CH}_{3} \rightarrow \mathrm{C}_{2} \mathrm{H}_{4}+\mathrm{H}_{2}
$$

followed by

$$
\mathrm{C}_{2} \mathrm{H}_{4} \stackrel{+\mathrm{H}}{\longrightarrow} \mathrm{C}_{2} \mathrm{H}_{3} \stackrel{+\mathrm{H}}{\longrightarrow} \mathrm{C}_{2} \mathrm{H}_{2} \stackrel{+\mathrm{H}}{\longrightarrow} \mathrm{C}_{2} \mathrm{H} \stackrel{+\mathrm{OH}}{\longrightarrow} \mathrm{CO}+\mathrm{CH}_{2}
$$

This sequence of reactions converts the second carbon atom released in reaction (4) into $\mathrm{CO}$. The intermediate products $\mathrm{CH}_{4}$ and $\mathrm{CH}_{3}$ accumulate to considerable abundances in the gas phase (cf. Fig. 1) since reaction (11) becomes fast only if the abundance of $\mathrm{CH}_{3}$ has increased to a sufficient level. This delays the conversion of the second carbon atom into $\mathrm{CO}$ to a temperature where already most of the carbon is gasified. 


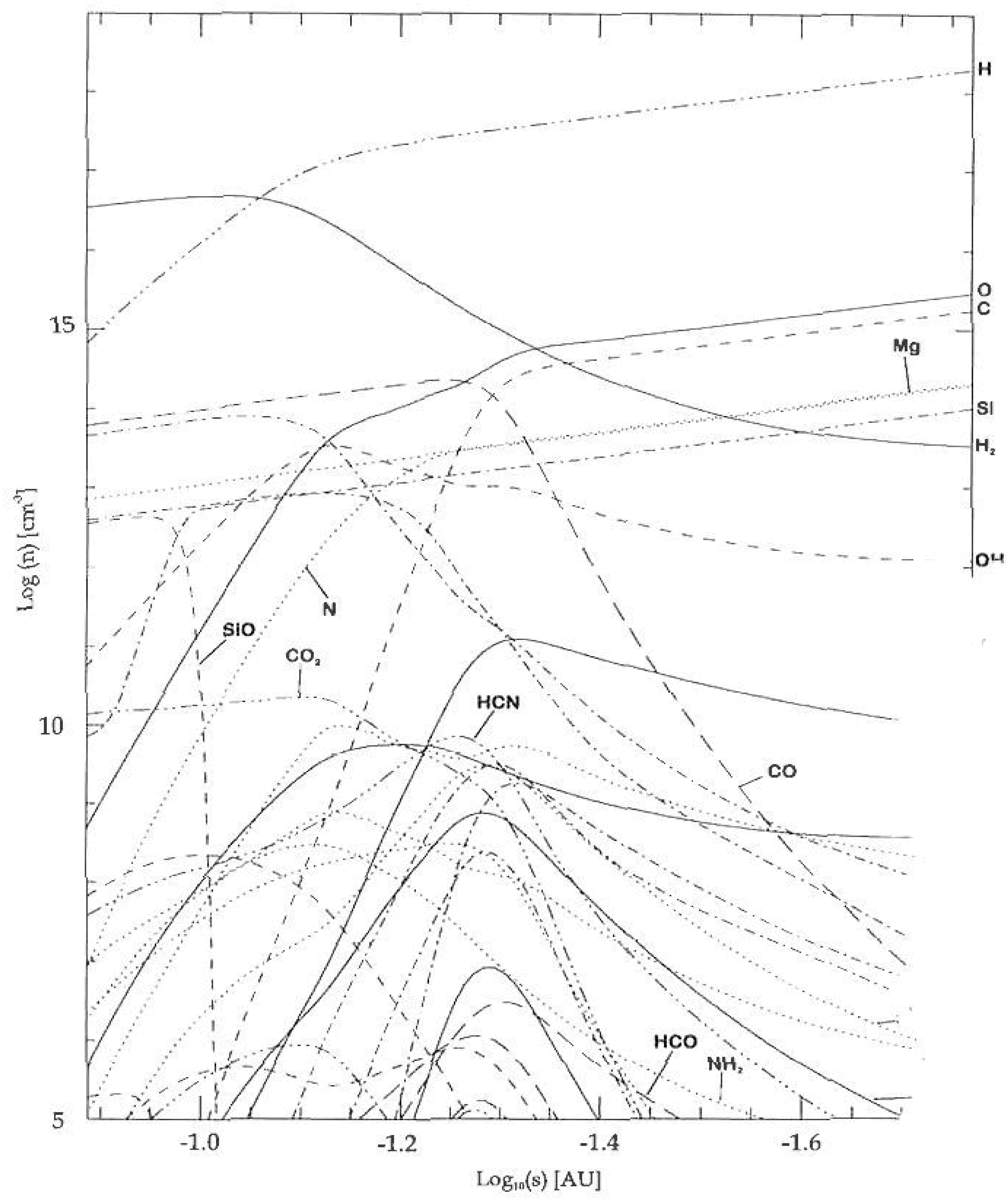

Figure 2. Chemical composition in the central plane of the disk: The ret $\mathrm{CO}$ dissociation.

An alternative path to $\mathrm{CO}$ would be reactions of $\mathrm{CH}_{i}$ molecules wit $\mathrm{OH}$ to form $\mathrm{HCO}$ or $\mathrm{H}_{2} \mathrm{CO}$ and from this $\mathrm{CO}$. These reaction paths turm. to be inefficient in the protoplanetary disk.

Between 0.5 and $0.1 \mathrm{AU}$ corresponding to the temperature regime $1650 \ldots 2100 \mathrm{~K}$ the olivine dust evaporates. This injects the decompon products $\mathrm{Mg}, \mathrm{SiO}$, and $\mathrm{O}$ into the gas phase. $\mathrm{SiO}$ is dissociated by $\mathrm{co}$ with $\mathrm{H}_{2}$ and $\mathrm{H}$. In our current calculations $\mathrm{Si}$ and $\mathrm{Mg}$ remain unchanged. 


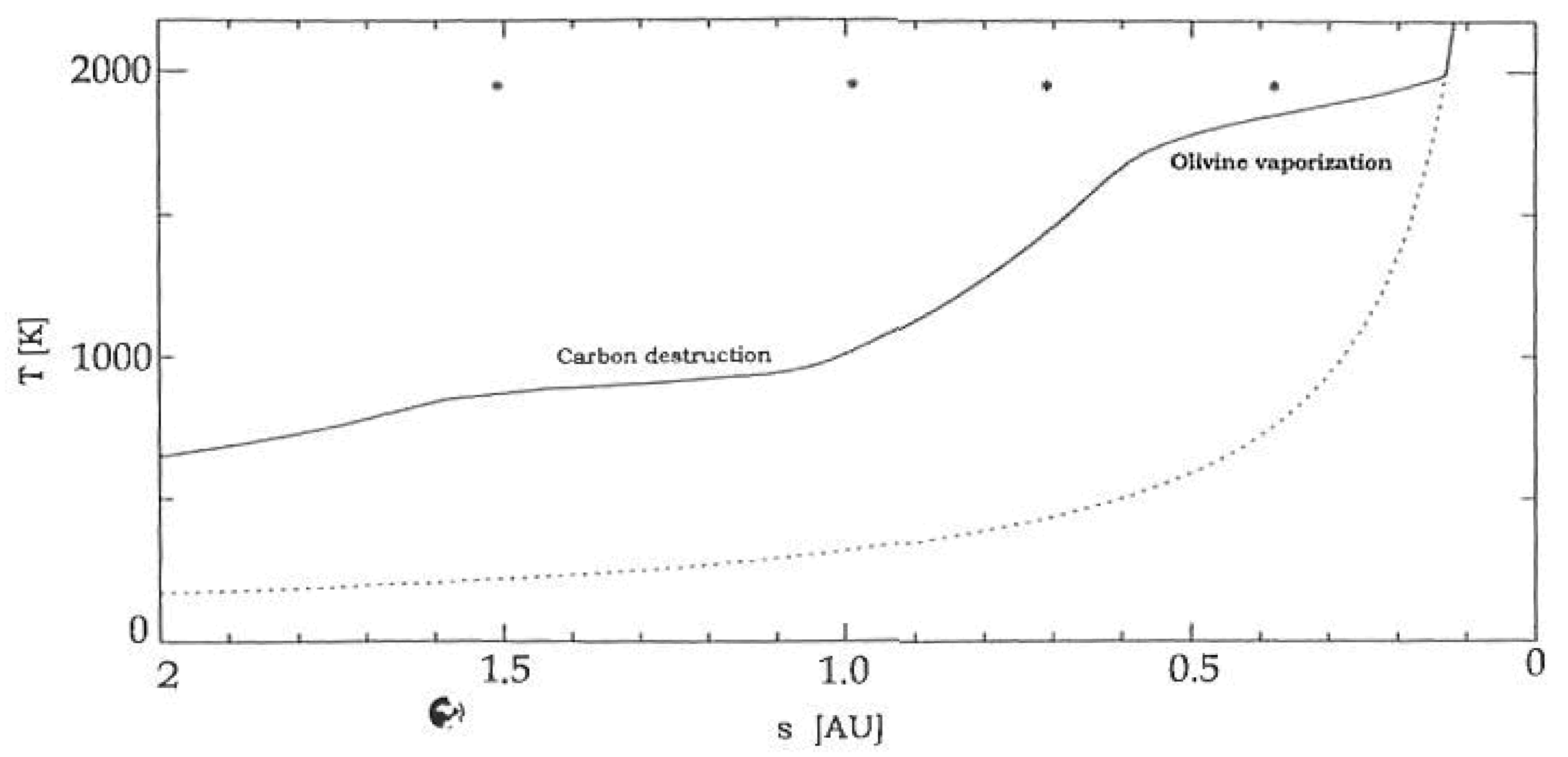

Figure 3 . Temperature in the central plane of the protoplanetary disk

ase because no reactions of these species are taken into account. The released by silicate decomposition forms $\mathrm{H}_{2} \mathrm{O}$.

tre 2 shows an enlargement of the inner part of the disk where a huge -f new molecular species occurs, especially carbon compounds. This is ie fact that part of the carbon is liberated by $\mathrm{CO}$ dissociation. Most rolecules dissociate for radii $s<0.06 \mathrm{AU}$. Dissociation of molecular " occurs at $s \approx 0.09 \mathrm{AU}$. Close to the center of the disk only the free $\mathrm{C}, \mathrm{N}, \mathrm{O}, \mathrm{Mg}$, and Si remain.

- direct consequence of the oxidation process for carbon dust, we obtain nethane density in the disk between 1.4 and 0.8 AU i.e. where the l planets are presently located. The earth is located in the middle part gion. This means that the earth has formed in an environment where Junts of organic molecules were present. We expect that additionally more complex organic molecules are formed in this region, as well, but lecules are not yet implemented in the present simulation. The carbon dation by $\mathrm{HCCO}$ formation in the surface reaction (4) is the key process 2. these huge amounts of methane. A test calculation which omits the leads to small amounts of methane and organics only.

interesting to compare the central plane temperature for two mod$\mathrm{h}$ and without considering dust opacities, see Fig. 3. The dotted line ponds to a model without dust opacity (i.e. only with an estimated gas tion coefficient of $0.01 \mathrm{~cm}^{2} / \mathrm{g}$ ). The temperature in a model with dust * (full line in Fig. 3) shows two plateaus corresponding to the evaporation on $(1-0.5 \mathrm{AU})$ and of olivine (0.5 - 0.1 AU). The plateaus are due to the se of opacity during dust destruction which counteracts a temperature with decreasing distance. The two curves merge where the last dust grains pear.

igure 4 shows the thickness of the accretion disk. The reduction of the ıess with decreasing absorption can be clearly seen. The figure additionally 


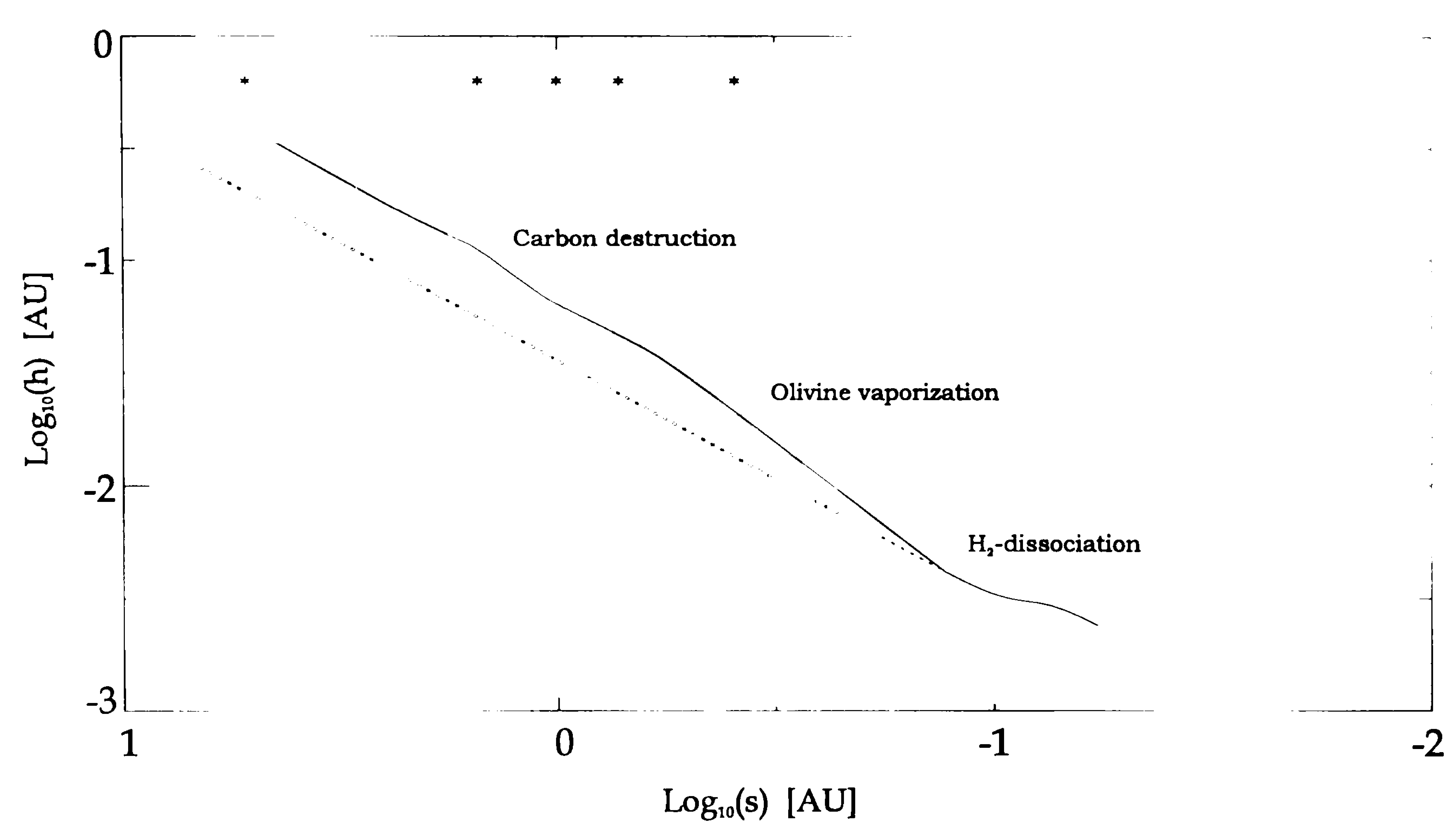

Figure 4. Thickness of the protoplanetary accretion disk

shows a short plateau in the disk around $s=0.05 \mathrm{AU}$ where $\mathrm{H}_{2}$ dissociates. The increasing particle density in this region prevents the further shrinkage of the disk over a short radius interval.

The asterisks in Figs. 3 and 4 indicate the present positions of the planets from Jupiter to Mercury.

Acknowledgments. This work has been supported by the Deutsche Forschungsgemeinschaft, Sonderforschungsbereich 359.

\section{References}

Anders, E., Grevesse, N., 1989, Geoch. and Cosmoch. Acta, 53, 197

Baulch, D.L., Cobos, C.J., Cox, R.A., Esser, C., Franck, P, Just, Th., Ker, J.A., Pilling, M.J., Troe, J., Walker, R.W., Warnatz, J., 1992, Jounal of physical and chemical reference data 21, 411

Bauer, I., Finocchi, F., Duschl, W.J., Gail, H.-P., Schlöder, J.P., 1996, A\&A (submitted)

Draine, B.T., 1985, ApJS 57, 587

Duschl, W.J., Gail, H.-P., Tscharnuter, W.M., 1996, A\&A, (in press)

El-Gamal, M., 1995, Thesis, University of Stuttgart

Mathis, J.S., Rumpl, W., Nordsieck, K.H., 1977, ApJ, 217, 425

Mitchell G.F., 1984, ApJS 54, 81

Pollack, J.B., Hollenbach, D., Beckwith, S., Simonelli, D.P., Roush, T., Fong, W., 1994, ApJ, 421, 615

Sandford, S.A. and Allamandola, L.J., 1993, ApJ, 417, 815 